\title{
SENSITIVITY OF DIGITAL ELECTRONIC WARFARE RECEIVERS
}

James Hedge, James B. Y. Tsui, David Sharpin

WRDC/AAWP-1, WPAFB

1. ABSTRACT

This paper will report the results of the study of the sensitivity of digital $\mathrm{EW}$ receivers. The best sensitivity one can expect from an $\mathrm{EW}$ receiver is achieved by designing the bandwidth of the receiver to match the pulse width of the input signals. Under this condition, the receiver can be considered as a matched filter. However, it is probably difficult to design an $\mathrm{EW}$ receiver which will perform as a matched filter to every input signal, although theoretically this can be done. This paper will provide an approach to improve the sensitivity of the receiver with reasonable complexity.

2. DATA OUTPUTS FROM A DIGITAL RECEIVER

Instead of starting with a theoretical approach, the outputs from a digital receiver will be examined

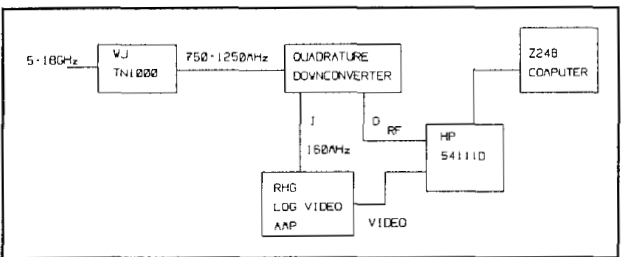

Figure 1 Experimental Setup

first. The experimental set-up is shown in Figure 1.

The first unit is an RF tuner with an input frequency range from .5 to $18 \mathrm{GHz}$ and an instantaneous bandwidth of $500 \mathrm{MHz}$. The output is from 750 to $1250 \mathrm{MHz}$. The second unit is a quadrature downconverter which converts the $750-1250 \mathrm{MHz}$ to $0-250 \mathrm{MHz}$ and provides both $I$ and 0 channels. The two channels are matched within $1 \mathrm{~dB}$ and 15 degrees across the operating bandwidth. At the I channel a $160 \mathrm{MHz}$ log amplifier with 40 $\mathrm{MHz}$ bandwidth was connected and the $\mathrm{Q}$ channel was connected to the input of an HP sampling scope.

The input signal frequency was set so that the output from the second converter was $160 \mathrm{MHz}$ which matched the operating frequency of the log amplifier. The amplitude of the input was adjusted until the video output from the log amplifier was at tangential sensitivity. The video output from the log amplifier was digitized and is shown in Figure $2 a$. The output from the $Q$ channel was also digitized and is shown in Figure $2 \mathrm{~b}$. Figure $2 \mathrm{~b}$ shows the effect of integration of the signal when the scope has infinite persistence. This allows the duration of the signal to be clearly seen. Figure $2 \mathrm{c}$ shows the data from a single digital scope sweep. It is
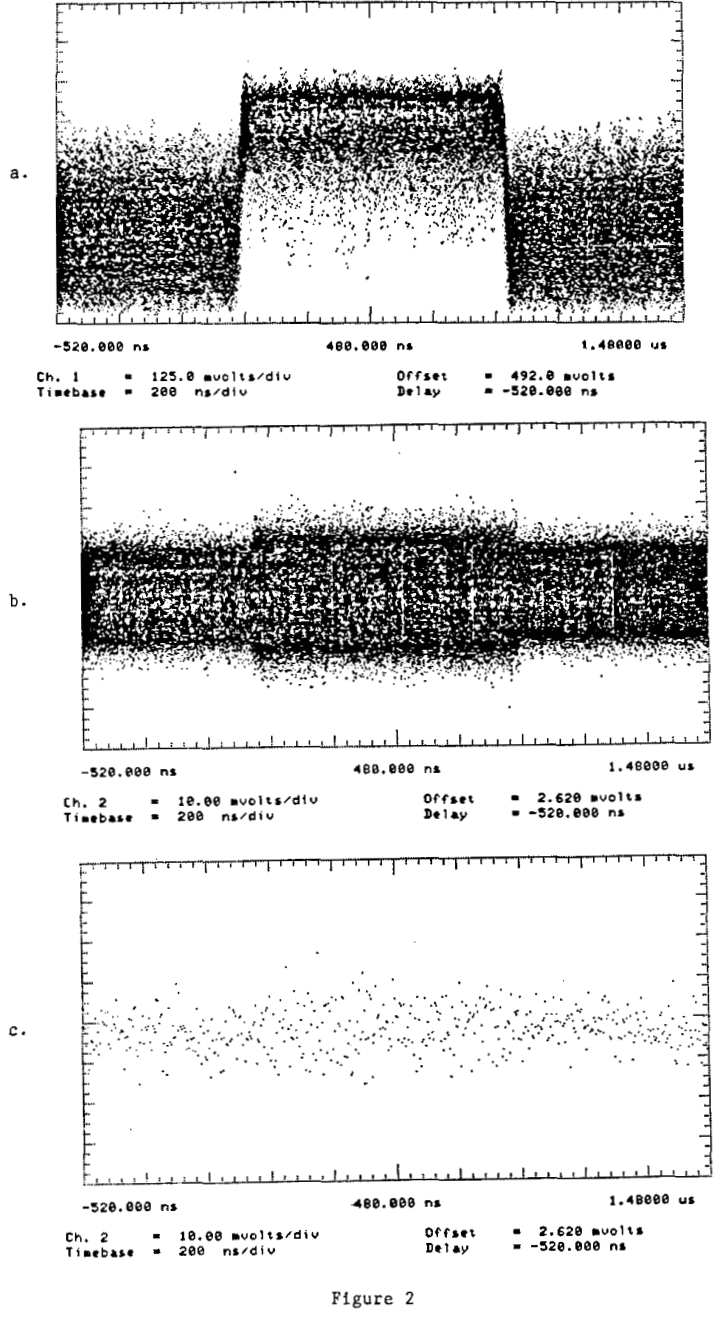

difficult to see the existence of the signal, therefore, it would be difficult to build a filter to match the bandwidth of the signal. It should be noted that the output level from Figure $2 b$ seems lower than at tangential sensitivity. The reason is that the log amplifier has less bandwidth ( $40 \mathrm{MHz})$ than the digital scope (250 $\mathrm{MHz})$.

3. IDEAL AND PRACTICAL APPROACHES The ideal approach to finding the signal is to perform a frequency analysis on the input data including only the portion that contains the input signal. In other words, one must know the leading and trailing edges of the input signal. Since from Figure $2 \mathrm{c}$ one does not know the leading and trailing edges of the signals, it is 
difficult to perform the ideal approach. Further, if the input data contains two pulses of different length, one has to perform the frequency analysis on the same data but with different starting and ending points to match the two different pulses. Obviously, although the ideal approach can be accomplished if enough processing is performed, it is, in general, not practical.

There are many different practical approaches to the analysis of the input data that would improve the sensitivity of the receiver. In this paper, one approach will be presented. This approach does not provide the best sensitivity that can be accomplished but it will provide an improvement over conventional EW receivers.

In this proposed approach, the Digital Fourier Transform (DFT) will be used to find the frequencies of the input signals. From the experimental data, one can see that in order to accomplish maximum sensitivity, the starting and ending data points must be known. In others words, the starting points and the length of the DFT must be determined. In this approach, several DFTs with fixed lengths are used. The length of the DFT can be related to the frequency resolution of through

$$
\delta f=\frac{2}{N \tau}
$$

where $\mathrm{N}$ is the total number of data points used in the DFT and $\tau$ is the time between sampling points. If one performs $64,256,1024$, and 2048 points DFTs with $\tau=1 \mathrm{~ns}$, the corresponding frequency resolutions will be 31.3 , $7.8,2$ and $1 \mathrm{MHz}$. Since the Nyquist sampling rate requires 2 points per cycle, the corresponding maximum instantaneous bandwidth of the system is $500 \mathrm{MHz}$. In order to limit the aliasing from the DFT, the input bandwidth is usually less than $500 \mathrm{MHz}$, say $250 \mathrm{MHz}$. From an analog receiver point of view, this approach is equivalent to building 4 channelized receivers, each with 250 $\mathrm{MHz}$ instantaneous bandwidth. The 4 receivers have frequency resolutions of $31.3,7.8,2$ and $1 \mathrm{MHz}$.

The next question is when to start processing the input data, and how the data should be overlapped. Different overlapping can be applied to the DFT. If there is zero overlapping, there is minimum processing required but there is less possibility of matching the input signals. More data overlapping requires more processing but also increase the possibility of matching the input signals to obtain better sensitivity. In this paper a sliding window will be used. In this approach, the input will shift by 32 data points each time. Thus, this approach will approximate the performance of a superhet receiver with a fixed bandwidth. In summary, this approach is equivalent to several channelized receivers each having a different resolution bandwidth.
4. DFT VERSUS FFT

In this last section, the digital Fourier transform

(DFT) is used rather than fast Fourier transform (FFT). The major differences between the two approaches are as follows: For general calculation of the Fourier transform in digital form, the well known fact is that the FFT is more efficient than the DFT. However for sliding operation, the DFT is more efficient than the FFT. This is because the new components can be calculated from the old ones. Assume that the input data is from $x_{0}, x_{1}, \ldots x_{N-1}$, and the mth component in the frequency domain is $x_{0,-1}(m)$. If one new point $x_{N}$ is obtained, the mth component from the data $\mathrm{x}_{1}$, $\mathrm{x}_{2}, \ldots$... $\mathrm{x}_{\mathrm{N}^{\prime}} \mathrm{x}_{1, N}(\mathrm{~m})$ can be

$x_{1, N}=\exp (j 2 \pi m / N)\left[x_{0, N-1}(m)+x_{N}-x_{0}\right]$

The major advantage of this equation is that the calculation is simpler than the FFT because one just needs to modify the previous values.

The FFT can be used to perform the initial calculation. For example, if 1024 data points will be used in the Fourier transform, it is reasonable to use the FFT to perform the initial calculation from $x_{0}$ to $x_{1023}$. If the DFT is used to calculate the initial Fourier transform, $\mathrm{N}^{2}$ operations will be required.

\section{FREOUENCY DETERMINATION}

Another difficult problem is to choose the peaks in the frequency domain. After the power spectrum is calculated in the frequency domain a threshold must be set such that the signal amplitude will cross it. One way to set the threshold is to take the average of all the frequency components. A frequency is then declared to be present when its amplitude is 5 times this average. This method was tested successfully.

The peaks must be evaluated at the

output of each DFT calculation. However, the peak component may not be consistent from one DFT output to the next. It may switch from one frequency component to its neighboring ones. This is true, particularly for the cases where the input frequency is between two adjacent frequency components. In order to take this problem into consideration one must allow comparison within limits. 
6. EXPERIMENTAL RESULTS

Some preliminary results were processed and the results will be presented here. The $I$ and $Q$ components of a signal were digitized at a $1 \mathrm{GHz}$ rate. The input was a pulsed signal with a center frequency of $284 \mathrm{MMHz}$, at $85 \mathrm{dbm}$ and a pulse width of about 250ns. The data collected in the time domain is shown in Figure $3 \mathrm{a}$ and $\mathrm{b}$. From these figures, it is difficult to detect the presence of the input signal. The program used to perform the DFT was the sliding FFT represented by Equation 2. The data from the I channel was used as the real part of the input signal and the $Q$ channel as the imaginary part. For simplicity, the frequency components from the DFT were not compared to select the center frequency of the signal. Instead, the known frequency component was calculated. The output of the selected frequency component was plotted as a function of time. The results are shown in Figures $3 \mathrm{c}$ through $3 \mathrm{~h}$. The difference in these figures is the length of the DFT.

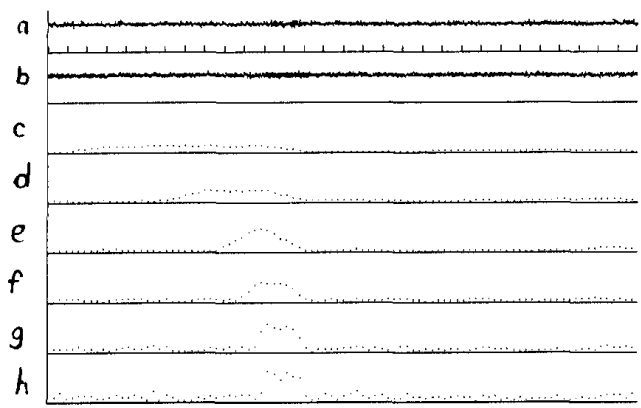

a. Output from I channel b. Output from O channel $\mathrm{C}$. Output from

1024 DFT d. Output from 512 DFT e. Output from 256 DFT $f$. Output from 128 DFT g. Output from 64 DFT $h$. Output from 32 DFT

Figure 3 Input and output of the DFT

since the data length in Figure $3 e$ corresponds to 256 ns which approximately matches the pulse width, the output has the highest signal-to-noise ratio. Under this condition, the frequency resolution bandwidth matches the signal bandwidth. As expected, when the resolution bandwidth of the receiver does not match the signal bandwidth, the signal-to-noise ratio deteriorates.

It appears that the output in Figure $3 \mathrm{f}$ has a higher signal- to-noise ratio than Figure $3 \mathrm{~d}$. If this result is true, or even if they have the same signal-to-noise ratio, it shows that it is possible to use shorter DFTs to detect the presence of a signal. It is desirable to use a shorter DFT, because it takes less calculation time. From these Figures, one can see that the outputs from the DFT can be considered as the correlation of two rectangular windows as shown in Figure 4. When the two windows have the same length, the
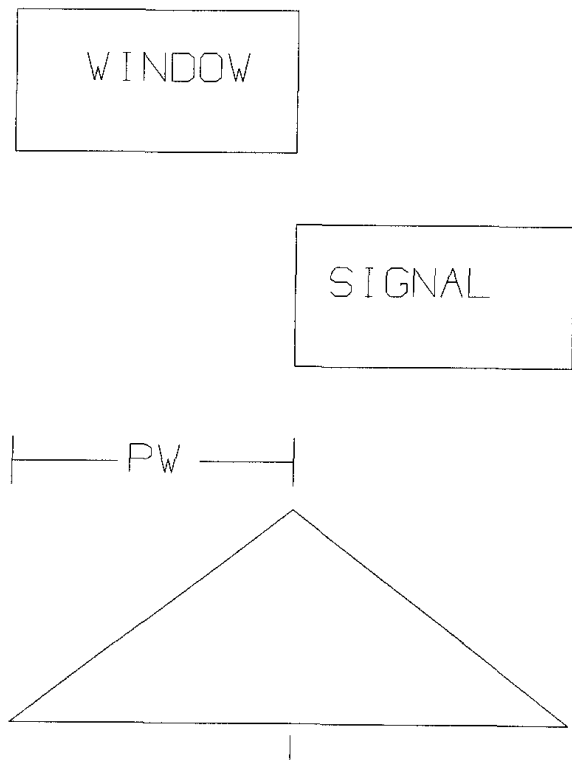

$T D A$

a. Window equal to sionol

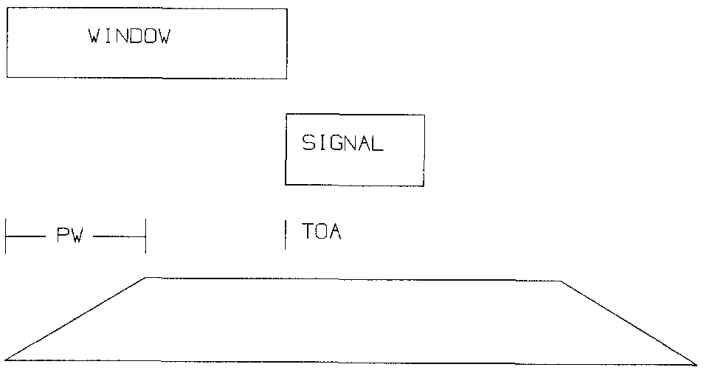

b. Window longer then sional 


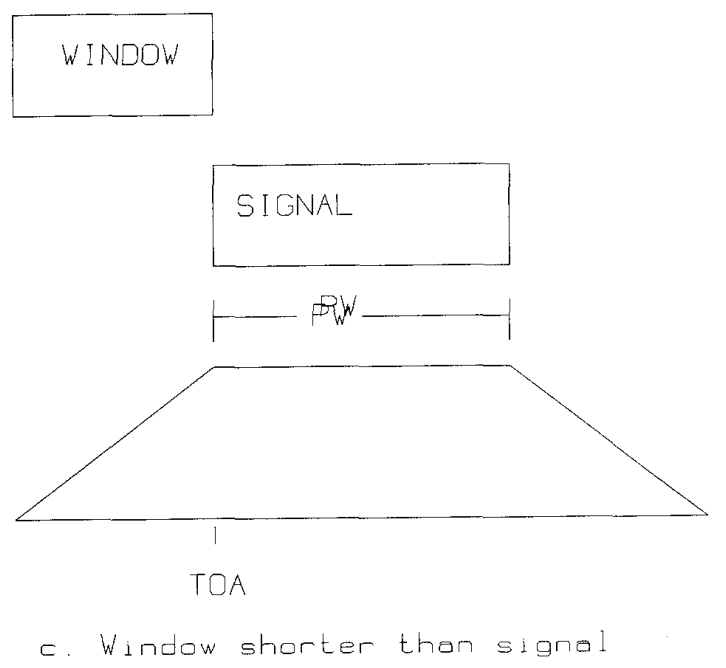

Figure 4 correlation of two rectangular windows

output is a triangle as shown in Figure 4a. If the window is longer than the input signal, the result is shown in Figure $4 \mathrm{~b}$. Figure $4 \mathrm{c}$ shows the result if the window is shorter than the input signal. Since the length of the window is known, the time of arrival (TOA) and pulse width (PW) can also be determined from the output of the DFT as shown in Figure 4 under different conditions.

7. SUMMARY

It has been demonstrated with actual digitized data that the sensitivity of a digital receiver can be improved if enough signal processing is applied. The approach presented can be considered as a brute force one. In order to obtain the optimum signal-to-noise ratio, excess processing is required. If some kind of adaptive signal processing can be developed, a wide band receiver with frequency resolution bandwidth depending on the signal length can be designed. This approach can improve the receiver sensitivity to match that of a specially designed superheterodyne receiver. One can apply this idea to figure 3, the TOA and PW can be obtained accordingly. However, when the signal-to-noise ratio is low, their obtained accuracy degrades.

References

1. Tom Springer. "Sliding FFT computes Frequency Spectra in Real Time," EDN Sept 29, 1988, ppl61-170 